Mathematical Modelling and Analysis

Volume 22 Number 2, March 2017, 252-269

https://doi.org/10.3846/13926292.2017.1292324

(C) Vilnius Gediminas Technical University, 2017
Publisher: Taylor\&Francis and VGTU

http://www.tandfonline.com/TMMA

ISSN: $1392-6292$

eISSN: $1648-3510$

\title{
Asymptotic Dynamics of a New Mechanochemical Model in Biological Patterns
}

\author{
Aibo Liu ${ }^{a}$ and Changchun Liu \\ ${ }^{a}$ School of Mathematics, Liaoning Normal University \\ 116029 Dalian, China \\ ${ }^{b}$ School of Mathematics, Jilin University \\ 130012 Changchun, China \\ E-mail(corresp.): liucc@jlu.edu.cn
}

Received September 14, 2016; revised January 21, 2017; published online March 1, 2017

\begin{abstract}
In this paper, we prove the existence of attractor for a new mechanochemical model with Neumann boundary conditions on a bounded domain of space dimension $n \leq 3$. Based on the regularity estimates for the semigroups and the classical existence theorem of global attractors, we prove that the mechanochemical model possesses a global attractor and $H^{k}$ attractor.
\end{abstract}

Keywords: mechanochemical model, biological patterns, attractor.

AMS Subject Classification: 35B41; 35K41; 92C15.

\section{Introduction}

In this paper, we study the following mechanochemical model

$$
\begin{aligned}
& \frac{\partial \phi}{\partial t}=\Delta \phi+\alpha \phi-\phi^{3}-\varepsilon \phi \psi, \\
& \frac{\partial \psi}{\partial t}=-\lambda(\Delta+1)^{2} \psi-\gamma \psi+g \psi^{2}-\psi^{3}-\frac{\varepsilon}{2} \phi^{2}
\end{aligned}
$$

for $(x, t) \in \Omega \times(0, \infty)$, where $\Omega \subset R^{n}(n \leq 3)$ is a bounded domain with smooth boundary, and all the parameters are arbitrarily given positive constants.

It was Morales, Rojas, Torres and Rubio [10] who first derived the system (1.1)-(1.2), which is to model ternary mixtures by using the theory of pattern formation and of polyelectrolytes, with mean-field approximations. Recently, Morales, Rojas, Oliveros and Hernández derived a new mechanochemical model based on Coupled Ginzburg-Landau and Swift-Hohenberg equations in biological patterns of marine animals [9]. The new model was proposed to describe some cellular interactions in three out layers of animal (such as the fish) marine skin. $\phi(x, t)$ represents the concentration difference of at least two pigment, and $\psi(x, t)$ is the difference of dermal cellular densities of at least two types of 
cells. The $\overrightarrow{J_{\phi}}=-\nabla \phi$ is the flux of pigment concentration difference $\phi$ in the epidermis, and $\overrightarrow{J_{\psi}}=2 \lambda \nabla \psi+\lambda \nabla \Delta \psi$ is the density of cell flux $\psi$ of the dermis. The system (1.1)-(1.2) is supplemented by the zero flux boundary condition,

$$
\overrightarrow{J_{\phi}} \cdot \nu=0, \quad \overrightarrow{J_{\psi}} \cdot \nu=0, \quad x \in \partial \Omega,
$$

where $\nu$ is the outward unit normal to $\partial \Omega$, that is

$$
\frac{\partial \phi}{\partial \nu}=0, \quad 2 \lambda \frac{\partial \psi}{\partial \nu}+\lambda \frac{\partial \Delta \psi}{\partial \nu}=0, \quad x \in \partial \Omega
$$

and the natural boundary condition

$$
\frac{\partial \psi}{\partial \nu}=0, \quad x \in \partial \Omega
$$

It follows from (b2) that (b1) can be replaced by

$$
\frac{\partial \Delta \psi}{\partial \nu}(x, t)=0, \quad x \in \partial \Omega .
$$

Hence, we consider the Neumann boundary conditions

$$
\frac{\partial \phi}{\partial \nu}(x, t)=\frac{\partial \Delta \psi}{\partial \nu}(x, t)=\frac{\partial \psi}{\partial \nu}(x, t)=0, \quad t>0, \quad x \in \partial \Omega
$$

and the initial condition

$$
\phi(x, 0)=\phi_{0}, \quad \psi(x, 0)=\psi_{0}, \quad x \in \Omega .
$$

The dynamic properties of the reaction-diffusion system (1.1)-(1.2), such as the global asymptotical behaviors of solutions and existence of global attractors are important. During the past years, many authors had paid much attention to the higher order equation $([1,4,6,7,16])$ or the reaction-diffusion systems $([2,3,11])$. You $([17,18,19])$ had proved the existence of global attractor for some Gray-Scott type systems. The main difficulties for treating the problem (1.1)-(1.2) are caused by the nonlinearity of low order terms, and linear higher order terms are not homogeneous. The source type nonlinear low terms and Neumann boundary conditions can not make us use Poincaré type inequality directly, thanks to strong absorptive terms $-\phi^{3}$ and $-\varphi^{3}$, which guarantees the existence of a global solution and will not blow up.

The paper is arranged as follows. In Section 2, some notations and the main results are stated. We present some estimates in Section 3, and then we prove that problem (1.1)-(1.4) possesses global attractors on $L^{2}(\Omega) \times H^{2}(\Omega)$ in Section 4. Based on this result, we prove the existence of global attractors for problem (1.1)-(1.4) in $H^{k}(k \geq 0)$ space in Section 5 .

\section{Statement of main results}

We first introduce the following abbreviations.

The notation $(\cdot, \cdot)$ for $L^{2}$-inner product will also be used for the notation of duality pairing between dual spaces, $\|\cdot\|=\|\cdot\|_{L^{2}}$. We use the same letter $C$ to denote different positive constants, and $C(\cdot, \cdot, \cdot)$ to denote positive constants depending on the quantities appearing in the parenthesis. 
Theorem 1. For any positive parameters $\alpha, \lambda, \gamma, g, \varepsilon$, any $\left(\phi_{0}, \psi_{0}\right)^{T} \in L^{2}(\Omega) \times$ $H^{2}(\Omega)$, and $n \leq 3$, there exists a global attractor $\mathcal{A}$ in the phase space $L^{2}(\Omega) \times$ $H^{2}(\Omega)$ for the solution semiflow $\{S(t)\}_{t \geq 0}$ on $L^{2}(\Omega) \times H^{2}(\Omega)$ generated by system (1.1)-(1.2) with the Neumann boundary conditions (1.3).

The basic theory of infinite dimensional dynamical systems and global attractors can be seen in $[11,15]$ and references therein. A few definitions are listed for clarity.

Definition 1. Let $\{S(t)\}_{t \geq 0}$ be a semiflow on a real Banach space $X$. A bounded subset $B_{0}$ of $X$ is called an absorbing set in $X$ for this semiflow, if for any bounded subset $B \subset X$ there is some finite time $t_{0} \geq 0$ depending on $B$ such that $S(t) B \subset B_{0}$ for all $t \geq t_{0}$.

Definition 2. Let $\{S(t)\}_{t \geq 0}$ be a semiflow on a real Banach space $X$ whose norm-induced metric is denoted by $d(\cdot, \cdot)$. A subset $\mathcal{A}$ of $X$ is called a global attractor for this semiflow, if the following properties are satisfied:

$(\mathrm{H} 1) \mathcal{A}$ is a nonempty, compact, invariant set in the sense that $S(t) \mathcal{A}=\mathcal{A}$ for any $t \geq 0$.

(H2) $\mathcal{A}$ attracts any bounded set $B$ of $X$ with respect to the Hausdorff distance,

$$
\operatorname{dist}(S(t) B, \mathcal{A})=\sup _{x \in B} \inf _{y \in \mathcal{A}} d(S(t) x, y) \rightarrow 0, \quad \text { as } \quad t \rightarrow \infty .
$$

Definition 3. A semiflow on a real Banach space $X$ is asymptotically compact if for any bounded sequence $u_{n}$ in $X$ and any sequence $t_{n} \subset(0, \infty)$ with $t_{n} \rightarrow$ $\infty$, there exist subsequences $u_{n_{k}}$ of $u_{n}$ and $t_{n_{k}}$ of $t_{n}$, such that $\lim _{k \rightarrow \infty} S\left(t_{n_{k}}\right) u_{n_{k}}$ exists in $X$.

Lemma 1. ( $[15])$ Let $\{S(t)\}_{t \geq 0}$ be a semiflow on a real Banach space $X$. If the following properties are satisfied:

(1) there exists a bounded absorbing set $B_{0} \subset X$ for $\{S(t)\}_{t \geq 0}$,

(2) $\{S(t)\}_{t \geq 0}$ is asymptotically compact on $X$,

then there exists a global attractor $\mathcal{A}$ for $\{S(t)\}_{t \geq 0}$ in $X$, which is given by

$$
\mathcal{A}=\omega\left(B_{0}\right):=\bigcap_{\tau \geq 0} C l_{X} \bigcup_{t \geq \tau}\left(S(t) B_{0}\right) .
$$

Let us write (1.1)-(1.2) as an evolution problem

$$
\left\{\begin{array}{l}
\frac{\partial u}{\partial t}+A u=F(u), \quad t>0 \\
u(0)=u_{0}
\end{array}\right.
$$

where $u=(\phi, \psi)^{T}, H:=L^{2}(\Omega) \times L^{2}(\Omega)$,

$$
\begin{aligned}
F(u) & =\left(\begin{array}{c}
F_{1}(\phi, \psi) \\
F_{2}(\phi, \psi)
\end{array}\right) \\
& =\left(\begin{array}{c}
\alpha \phi-\phi^{3}-\varepsilon \phi \psi \\
-2 \lambda \Delta \psi-(\gamma+\lambda) \psi+g \psi^{2}-\psi^{3}-\frac{\varepsilon}{2} \phi^{2}
\end{array}\right): D\left(A^{\frac{1}{2}}\right) \rightarrow H
\end{aligned}
$$


and

$$
A=\left(\begin{array}{rl}
-\Delta & 0 \\
0 & \lambda \Delta^{2}
\end{array}\right): D(A) \rightarrow H .
$$

$F(u)$ and the operator $A$ is considered on the Hilbert space $L^{2}$ with dense domain

$$
\begin{aligned}
& D(A)=\left\{u \in H^{2}(\Omega) \times H^{4}(\Omega): \frac{\partial \phi}{\partial \nu}=\frac{\partial \Delta \psi}{\partial \nu}=\frac{\partial \psi}{\partial \nu}=0, \text { on } \partial \Omega\right\} \\
& D\left(A^{\frac{1}{2}}\right)=\left\{u \in H^{1}(\Omega) \times H^{2}(\Omega): \frac{\partial \phi}{\partial \nu}=\frac{\partial \psi}{\partial \nu}=0, \text { on } \partial \Omega\right\} .
\end{aligned}
$$

Let

$$
\begin{aligned}
& E:=\left\{u \in H^{1}(\Omega) \times H^{1}(\Omega): \frac{\partial \phi}{\partial \nu}=\frac{\partial \psi}{\partial \nu}=0, \text { on } \partial \Omega\right\}, \\
& E_{1}:=\left\{u \in L^{2}(\Omega) \times H^{2}(\Omega): \frac{\partial \psi}{\partial \nu}=0, \text { on } \partial \Omega\right\}, \\
& E_{2}:=\left\{u \in H^{1}(\Omega) \times H^{3}(\Omega): \frac{\partial \phi}{\partial \nu}=\frac{\partial \psi}{\partial \nu}=\frac{\partial \Delta \psi}{\partial \nu}=0, \text { on } \partial \Omega\right\} .
\end{aligned}
$$

In order to prove the existence of solutions, we shall show $A$ is sectorial, $F(u)$ is locally Lipschitz continuous as the operation between the space $D\left(A^{1 / 2}\right)$ and $L^{2} \times L^{2}$, denoting by $\langle\cdot, \cdot\rangle$ the scalar product in $L^{2}$ and

$$
T:=A+\delta_{0} \Gamma, \quad \Gamma:=\left(\begin{array}{ll}
1 & 0 \\
0 & 1
\end{array}\right) .
$$

where $\delta_{0}>0$ is taken sufficient large. For any $u=(\phi, \psi)^{T}, v=(\bar{\phi}, \bar{\psi})^{T}$, $\{u, v\} \in D(A)$, noticing $\Gamma$ is symmetric we find that

$$
\begin{aligned}
\langle T u, v\rangle & =\int_{\Omega}\left(-\Delta \phi \bar{\phi}+\lambda \Delta^{2} \psi \bar{\psi}\right) d x+\int_{\Omega} \delta_{0} u^{T} \Gamma v d x \\
& =\int_{\Omega}(\nabla \phi \nabla \bar{\phi}+\lambda \Delta \psi \Delta \bar{\psi}) d x \int_{\Omega} \delta_{0} u^{T} \Gamma v d x \\
& =\int_{\Omega}\left(-\phi \Delta \bar{\phi}+\psi \lambda \Delta^{2} \bar{\psi}\right) d x+\int_{\Omega} \delta_{0} v^{T} \Gamma u d x=\langle u, T v\rangle
\end{aligned}
$$

which proves the symmetry of $A$. Next, since $\Gamma$ is positive definite, the operator $A$ is bounded below, that is, for each $u \in D(A)$,

$$
\begin{aligned}
\langle T u, u\rangle & =\int_{\Omega}\left(-\Delta \phi \phi+\lambda \Delta^{2} \psi \psi\right) d x+\int_{\Omega} \delta_{0} u^{T} \Gamma u d x \\
& =\int_{\Omega}\left((\nabla \phi)^{2}+\lambda(\Delta \psi)^{2}\right) d x+\int_{\Omega} \delta_{0} u^{T} \Gamma u d x \geq \delta_{0}\langle u, u\rangle
\end{aligned}
$$

$A$ is self-adjoint and bounded below, which means that $A$ is itself sectorial. We prove the local Lipschitz continuity of nonlinear function $F(u)$

$$
\forall u, v \in U \subset D\left(A^{1 / 2}\right), \quad\|F(u)-F(v)\|_{L^{2}} \leq K_{U}\|u-v\|_{D\left(A^{1 / 2}\right)} .
$$


Notice $H^{2} \hookrightarrow L^{\infty}$ and $H^{1} \hookrightarrow L^{6}$ for $n \leq 3$.

We find by differentiation that, for $\forall u, v \in U \subset D\left(A^{1 / 2}\right)$

$$
\begin{aligned}
& \left\|F_{1}(u)-F_{1}(v)\right\|_{L^{2}} \\
& \quad=\left\|\left(\alpha-\phi^{2}-\phi \bar{\phi}-\bar{\phi}^{2}\right)(\phi-\bar{\phi})+\varepsilon \psi(\phi-\bar{\phi})+\varepsilon \bar{\phi}(\psi-\bar{\psi})\right\|_{L^{2}} \\
& \quad \leq\left\|\left(\alpha-\phi^{2}-\phi \bar{\phi}-\bar{\phi}^{2}\right)(\phi-\bar{\phi})\right\|_{L^{2}}+\|\varepsilon \psi(\phi-\bar{\phi})\|_{L^{2}}+\|\varepsilon \bar{\phi}(\psi-\bar{\psi})\|_{L^{2}} \\
& \quad \leq C\left(|\alpha|+\|\bar{\phi}\|_{L^{6}}^{2}+\|\phi\|_{L^{6}}^{2}+\|\psi\|_{L^{\infty}}\right)\|\phi-\bar{\phi}\|_{L^{6}}+\varepsilon\|\bar{\phi}\|_{L^{2}}\|\psi-\bar{\psi}\|_{L^{\infty}} \\
& \quad \leq C\|\phi-\bar{\phi}\|_{H^{1}}+C\|\psi-\bar{\psi}\|_{H^{2}} \leq K_{U}\|u-v\|_{D\left(A^{1 / 2}\right)}
\end{aligned}
$$

and

$$
\begin{aligned}
\| F_{2}(u) & -F_{2}(v)\left\|_{L^{2}}=\right\|-2 \lambda \Delta(\psi-\bar{\psi})-(\gamma+\lambda)(\psi-\bar{\psi})+g(\psi+\bar{\psi})(\psi-\bar{\psi}) \\
& +\left(-\psi^{2}-\psi \bar{\psi}-\bar{\psi}^{2}\right)(\psi-\bar{\psi})-\frac{\varepsilon}{2}(\phi+\bar{\phi})(\phi-\bar{\phi}) \|_{L^{2}} \\
\leq C & \left(\|\bar{\psi}\|_{L^{6}}^{2}+\|\psi\|_{L^{6}}^{2}\right)\|\phi-\bar{\psi}\|_{L^{6}}+2 \lambda\|\psi-\bar{\psi}\|_{H^{2}} \\
& +C\left(\|\phi\|_{L^{4}}^{2}+\|\bar{\phi}\|_{L^{4}}^{2}\right)\|\phi-\bar{\phi}\|_{L^{4}} \leq K_{U}\|u-v\|_{D\left(A^{1 / 2}\right)} .
\end{aligned}
$$

The general theory of ( [5]) guarantees the existence of a local solution for system (1.1)-(1.2). The priori estimate Lemma 2-Lemma 5 implying the local solution can be a global one for $t$. Moreover, the family of operators $S(t)_{t \geq 0}$ forms a strongly continuous semigroup on the space $D\left(A^{1 / 2}\right)$, which has the property

$u \in C\left(\left[0, T_{\max }\right) ; H^{1} \times H^{2}\right) \cap C^{1}\left(\left(0, T_{\max }\right) ; H^{1} \times H^{2}\right) \cap L^{2}\left(\left[0, T_{\max }\right) ; L^{2} \times L^{2}\right)$.

\section{Absorbing sets}

Lemma 2. For any given $R>0$ there exists a constant $M_{1}(R)>0$ such that if the initial data $u_{0}=\left(\phi_{0}, \psi_{0}\right)^{T} \in H$ and $\left\|u_{0}\right\|_{H}^{2} \leq R$, then $S(t) u_{0}=(\phi, \psi)^{T} \in H$ for all $t \geq 0$, and

$$
\left\|S(t) u_{0}\right\|_{H}^{2} \leq M_{1}(R), \quad \text { for } \quad t \geq 0 .
$$

Proof. Taking the inner products $\langle(1.1), 2 \phi\rangle$, and $\langle(1.2), 2 \psi\rangle$, then summing up the resulting equalities and by the Neumann boundary conditions, we get

$$
\begin{aligned}
& \frac{d}{d t}\left(\|\phi\|^{2}+\|\psi\|^{2}\right)+2\|\nabla \phi\|^{2}+2 \lambda \int_{\Omega}[(\Delta+1) \psi]^{2} d x \\
& \quad=2 \alpha\|\phi\|^{2}+2 g \int_{\Omega} \psi^{3} d x-3 \varepsilon \int_{\Omega} \phi^{2} \psi d x-2\|\phi\|_{L^{4}}^{4}-2\|\psi\|_{L^{4}}^{4}-2 \gamma\|\psi\|^{2},
\end{aligned}
$$

that is

$$
\begin{aligned}
& \frac{d}{d t}\left(\|\phi\|^{2}+\|\psi\|^{2}\right)+\kappa\left(\|\phi\|^{2}+\|\psi\|^{2}\right)+2\|\nabla \phi\|^{2}+2 \lambda \int[(\Delta+1) \psi]^{2} d x \\
& \quad \leq 2 \Lambda(\phi, \psi),
\end{aligned}
$$


where

$$
\begin{aligned}
\Lambda(\phi, \psi)=- & \int\left(\frac{\sqrt{2}}{2} \phi^{2}-\frac{3 \sqrt{2}}{4} \psi\right)^{2} d x+\left(\frac{9 \varepsilon^{2}}{8}+\frac{\kappa}{2}\right)\|\psi\|^{2}+\frac{\kappa}{2}\|\phi\|^{2}-\frac{1}{2}\|\phi\|_{L^{4}}^{4} \\
& -\gamma\|\psi\|^{2}+g\|\psi\|_{L^{3}}^{3}-\|\psi\|_{L^{4}}^{4}
\end{aligned}
$$

The Young inequalities yield

$$
\begin{aligned}
& \frac{\kappa}{2}\|\phi\|^{2} \leq \frac{\kappa \varepsilon_{1}}{4}\|\phi\|_{L^{4}}^{4}+\frac{\kappa}{4 \varepsilon_{1}}|\Omega|, \quad g\|\psi\|_{L^{3}}^{3} \leq g \varepsilon_{2}\|\psi\|_{L^{4}}^{4}+g \varepsilon_{2}^{-3}|\Omega|, \\
& \left(\frac{9 \varepsilon^{2}}{8}+\frac{\kappa}{2}\right)\|\psi\|^{2} \leq\left(\frac{9 \varepsilon^{2}}{8}+\frac{\kappa}{2}\right) \frac{\varepsilon_{3}}{2}\|\psi\|_{L^{4}}^{4}+\left(\frac{9 \varepsilon^{2}}{8}+\frac{\kappa}{2}\right) \frac{1}{2 \varepsilon_{3}}|\Omega|,
\end{aligned}
$$

where $\varepsilon_{1}, \varepsilon_{2}, \varepsilon_{3}$ are arbitrarily positive constants. Hence, we have

$$
\begin{aligned}
\Lambda(\phi, \psi) & \leq\left(g \varepsilon_{2}+\left(\frac{9 \varepsilon^{2}}{8}+\frac{\kappa}{2}\right) \frac{\varepsilon_{3}}{2}-1\right)\|\psi\|_{L^{4}}^{4}-\int\left(\frac{\sqrt{2}}{2} \phi^{2}-\frac{3 \sqrt{2}}{4} \psi\right)^{2} d x \\
& -\gamma\|\psi\|^{2}+\left(\frac{\kappa \varepsilon_{1}}{4}-\frac{1}{2}\right)\|\phi\|_{L^{4}}^{4}+\left(\frac{\kappa}{4 \varepsilon_{1}}+g \varepsilon_{2}^{-3}+\left(\frac{9 \varepsilon^{2}}{8}+\frac{\kappa}{2}\right) \frac{1}{2 \varepsilon_{3}}\right)|\Omega| .
\end{aligned}
$$

We take $\varepsilon_{1}=\frac{1}{2}, \varepsilon_{2}=\frac{1}{2 g}, \varepsilon_{3}=\frac{1}{\left(9 \varepsilon^{2} / 8+\kappa / 2\right)}$ and $0<\kappa \leq 2$, then

$$
\Lambda(\phi, \psi) \leq\left(\frac{\kappa}{4 \varepsilon_{1}}+g \varepsilon_{2}^{-3}+\left(\frac{9 \varepsilon^{2}}{8}+\frac{\kappa}{2}\right) \frac{1}{2 \varepsilon_{3}}\right)|\Omega| \equiv \frac{1}{2} C^{*}(\kappa, g, \varepsilon,|\Omega|) .
$$

From (3.1) and (3.2), we obtain

$$
\frac{d}{d t}\left(\|\phi\|^{2}+\|\psi\|^{2}\right)+\kappa\left(\|\phi\|^{2}+\|\psi\|^{2}\right) \leq C^{*}(\kappa, g, \varepsilon,|\Omega|), \quad \text { for } t \in\left[0, T_{\max }\right)
$$

then, applying the Gronwall inequality, we deduce that

$$
\left(\|\phi\|^{2}+\|\psi\|^{2}\right) \leq e^{-\kappa t}\left(\left\|\phi_{0}\right\|^{2}+\left\|\psi_{0}\right\|^{2}\right)+\frac{C^{*}}{\kappa}, \quad \text { for } \quad t \in\left[0, T_{\max }\right) .
$$

Let $M_{1}$ be the constant $M_{1}=\frac{C^{*}}{\kappa}+1$. The proof is completed.

Lemma 3. For any given $R>0$ there exists a constant $M_{2}(R)>0$ such that if the initial data $u_{0}=\left(\phi_{0}, \psi_{0}\right)^{T} \in E$ and $\left\|u_{0}\right\|_{E}^{2} \leq R$, then $S(t) u_{0}=(\phi, \psi)^{T} \in E$ for all $t \geq 0$, and

$$
\left\|S(t) u_{0}\right\|_{E}^{2} \leq M_{2}(R), \quad \text { for } \quad t \geq 0
$$

Proof. Take the inner products $\langle(1.1),-2 \Delta \phi\rangle$, and $\langle(1.2),-2 \Delta \psi\rangle$. Then sum up the resulting equalities

$$
\begin{aligned}
& \frac{d}{d t}\left(\|\nabla \phi\|^{2}+\|\nabla \psi\|^{2}\right)=-2\|\Delta \phi\|^{2}-6 \int_{\Omega} \phi^{2}(\nabla \phi)^{2} d x+2 \alpha\|\nabla \phi\|^{2} \\
& \quad-2\|\nabla \Delta \psi\|^{2}-2(\gamma+\lambda)\|\nabla \psi\|^{2}-6 \int_{\Omega} \psi^{2}(\nabla \psi)^{2} d x+4 g \int_{\Omega} \psi(\nabla \psi)^{2} d x \\
& \quad+4 \lambda\|\Delta \psi\|^{2}-2 \varepsilon \int_{\Omega} \phi \nabla \phi \nabla \psi d x+2 \varepsilon \int_{\Omega} \psi \phi \Delta \phi d x
\end{aligned}
$$


that is

$$
\frac{d}{d t}\left(\|\nabla \phi\|^{2}+\|\nabla \psi\|^{2}\right)+\kappa_{2}\left(\|\nabla \phi\|^{2}+\|\nabla \psi\|^{2}\right) \equiv \Lambda_{2}(\phi, \psi),
$$

where

$$
\begin{aligned}
\Lambda_{2}(\phi, \psi)= & -\int_{\Omega}\left(\sqrt{6} \phi \nabla \phi+\frac{\varepsilon \nabla \psi}{\sqrt{6}}\right)^{2} d x-\int_{\Omega}\left(\sqrt{6} \psi \nabla \psi+\frac{\sqrt{6}}{3} g \nabla \psi\right)^{2} d x \\
& +\left(2 \alpha+\kappa_{2}\right)\|\nabla \phi\|^{2}+\left(\kappa_{2}+\frac{\varepsilon^{2}}{6}+\frac{2}{3} g^{2}-2 \gamma-2 \lambda\right)\|\nabla \psi\|^{2} \\
& -2\|\Delta \phi\|^{2}-2 \lambda\|\nabla \Delta \psi\|^{2}+2 \varepsilon \int_{\Omega} \psi \phi \Delta \phi d x+4 \lambda\|\Delta \psi\|^{2} .
\end{aligned}
$$

Then, applying the Nirenberg inequality $(n \leq 3)$, we deduce that

$$
\begin{aligned}
& \|\Delta \psi\| \leq C_{1}\|\nabla \Delta \psi\|^{1 / 2}\|\psi\|^{1 / 2}+C_{2}\|\psi\|, \\
& \|\phi\|_{L^{4}} \leq C_{1}\|\Delta \phi\|^{3 / 8}\|\phi\|^{5 / 8}+C_{2}\|\phi\|, \\
& \|\psi\|_{L^{4}} \leq C_{1}\|\nabla \Delta \psi\|^{1 / 4}\|\psi\|^{3 / 4}+C_{2}\|\psi\|, \\
& \|\nabla \phi\| \leq C_{1}\|\Delta \phi\|^{1 / 2}\|\phi\|^{1 / 2}+C_{2}\|\phi\|, \\
& \|\nabla \psi\| \leq C_{1}\|\nabla \Delta \psi\|^{1 / 3}\|\psi\|^{2 / 3}+C_{2}\|\psi\| .
\end{aligned}
$$

Using the Young inequality and Lemma 2, we have

$$
\begin{aligned}
& 2 \varepsilon \int_{\Omega} \psi \phi \Delta \phi d x \leq \frac{1}{4}\|\Delta \phi\|^{2}+C(\varepsilon)\|\phi\|_{L^{4}}^{4}+C(\varepsilon)\|\psi\|_{L^{4}}^{4} \\
& \leq \frac{1}{2}\|\Delta \phi\|^{2}+\frac{\lambda}{2}\|\nabla \Delta \psi\|^{2}+C(\varepsilon), \\
& 4 \lambda\|\Delta \psi\|^{2} \leq \frac{\lambda}{4}\|\nabla \Delta \psi\|^{2}+C(\lambda), \quad\left(2 \alpha+\kappa_{2}\right)\|\nabla \phi\|^{2} \leq \frac{1}{2}\|\Delta \phi\|^{2}+C\left(\alpha, \kappa_{2}\right)
\end{aligned}
$$

and

$$
\left(\kappa_{2}+\frac{\varepsilon^{2}}{6}+\frac{2}{3} g^{2}-2 \gamma-2 \lambda\right)\|\nabla \psi\|^{2} \leq \frac{\lambda}{4}\|\nabla \Delta \psi\|^{2}+C\left(\kappa_{2}, \varepsilon, g, \gamma, \lambda\right) .
$$

Summing up the resulting equalities, we have

$$
\begin{aligned}
\Lambda_{2}(\phi, \psi) \leq & -\int_{\Omega}\left(\sqrt{6} \phi \nabla \phi+\frac{\varepsilon \nabla \psi}{\sqrt{6}}\right)^{2} d x-\int_{\Omega}\left(\sqrt{6} \psi \nabla \psi+\frac{\sqrt{6}}{3} g \nabla \psi\right)^{2} d x \\
& -\|\Delta \phi\|^{2}-\lambda\|\nabla \Delta \psi\|^{2}+C_{2}^{*}\left(\alpha, \gamma, g, \lambda, \kappa_{2}, \varepsilon,|\Omega|\right) .
\end{aligned}
$$

From (3.3) and (3.4), we find that

$$
\frac{d}{d t}\left(\|\nabla \phi\|^{2}+\|\nabla \psi\|^{2}\right)+\kappa_{2}\left(\|\nabla \phi\|^{2}+\|\nabla \psi\|^{2}\right) \leq C_{2}^{*}\left(\alpha, \gamma, g, \lambda, \kappa_{2}, \varepsilon,|\Omega|\right),
$$


then, applying the Gronwall inequality, we deduce that

$$
\left(\|\nabla \phi\|^{2}+\|\nabla \psi\|^{2}\right) \leq e^{-\kappa_{2} t}\left(\left\|\nabla \phi_{0}\right\|^{2}+\left\|\nabla \psi_{0}\right\|^{2}\right)+\frac{C_{2}^{*}}{\kappa_{2}}, \text { for } t \in\left[0, T_{\max }\right) .
$$

Let $M_{2}$ be the constant $M_{2}=\frac{C_{2}^{*}}{\kappa_{2}}+1$. The proof is completed.

Lemma 4. For any given $R>0$ there exists a constant $M_{3}(R)>0$ such that if the initial data $u_{0}=\left(\phi_{0}, \psi_{0}\right)^{T} \in E_{1}$ and $\left\|u_{0}\right\|_{E_{1}} \leq R$, then $S(t) u_{0}=(\phi, \psi)^{T} \in$ $E_{1}$ for all $t \geq 0$, and

$$
\left\|S(t) u_{0}\right\|_{E}^{2} \leq M_{3}(R), \quad \text { for } \quad t \geq 0
$$

Proof. Taking the inner products $\langle(1.1), 2 \phi\rangle$, and $\left\langle(1.2), 2 \Delta^{2} \psi\right\rangle$, by the Neumann boundary conditions, we get

$$
\begin{aligned}
& \frac{d}{d t}\|\phi\|^{2}=-2\|\nabla \phi\|^{2}+2 \alpha\|\phi\|^{2}-2\|\phi\|_{L^{4}}^{4}-2 \varepsilon \int_{\Omega} \phi^{2} \psi d x, \\
& \frac{d}{d t}\|\Delta \psi\|^{2}=-2 \lambda\left\|\Delta^{2} \psi\right\|^{2}+4 \lambda\|\nabla \Delta \psi\|^{2}-2(\lambda+\gamma)\|\Delta \psi\|^{2} \\
& \quad+2 \alpha \int_{\Omega} \psi^{2} \Delta^{2} \psi d x-2 \int_{\Omega} \psi^{3} \Delta^{2} \psi d x-\varepsilon \int_{\Omega} \phi^{2} \Delta^{2} \psi d x
\end{aligned}
$$

Then summing up the resulting equalities, we see that

$$
\begin{aligned}
& \frac{d}{d t}\left(\|\phi\|^{2}+\|\Delta \psi\|^{2}\right)+\kappa_{3}\left(\|\phi\|^{2}+\|\Delta \psi\|^{2}\right) \\
= & -2\|\nabla \phi\|^{2}+\left(2 \alpha+\kappa_{3}\right)\|\phi\|^{2}-2\|\phi\|_{L^{4}}^{4}-2 \varepsilon \int_{\Omega} \phi^{2} \psi d x-2 \lambda\left\|\Delta^{2} \psi\right\|^{2} \\
& +4 \lambda\|\nabla \Delta \psi\|^{2}-2\left(\lambda+\gamma-\frac{\kappa_{3}}{2}\right)\|\Delta \psi\|^{2}+2 \alpha \int_{\Omega} \psi^{2} \Delta^{2} \psi d x \\
& -2 \int_{\Omega} \psi^{3} \Delta^{2} \psi d x-\varepsilon \int_{\Omega} \phi^{2} \Delta^{2} \psi d x \equiv \Lambda_{3}(\phi, \psi) .
\end{aligned}
$$

By the Nirenberg inequalities, we get

$$
\begin{aligned}
& \|\phi\|_{L^{4}}^{4} \leq C_{1}\|\nabla \phi\|^{3 / 4}\|\phi\|^{1 / 4}+C_{2}\|\phi\|, \\
& \|\nabla \Delta \psi\| \leq C_{1}\left\|\Delta^{2} \psi\right\|^{3 / 4}\|\psi\|^{1 / 4}+C_{2}\|\psi\|, \\
& \|\psi\|_{L^{4}} \leq C_{1}\left\|\Delta^{2} \psi\right\|^{3 / 16}\|\psi\|^{13 / 16}+C_{2}\|\psi\|, \\
& \|\psi\|_{L^{6}} \leq C_{1}\left\|\Delta^{2} \psi\right\|^{1 / 4}\|\psi\|^{3 / 4}+C_{2}\|\psi\| .
\end{aligned}
$$

Using the Young inequalities, Lemma 2 and above inequalities, we obtain

$$
\begin{aligned}
& 2 \varepsilon \int_{\Omega} \phi^{2} \psi d x \leq\|\phi\|_{L^{4}}^{4}+\varepsilon\|\psi\|^{2} \leq\|\nabla \phi\|^{2}+C(\varepsilon) \\
& 2 \alpha \int_{\Omega} \psi^{2} \Delta^{2} \psi d x \leq \frac{\lambda}{4}\left\|\Delta^{2} \psi\right\|^{2}+C(\lambda, \alpha)\|\psi\|_{L^{4}}^{4} \leq \frac{\lambda}{2}\left\|\Delta^{2} \psi\right\|^{2}+C(\lambda, \alpha,|\Omega|)
\end{aligned}
$$


and

$$
2 \int_{\Omega} \psi^{3} \Delta^{2} \psi d x \leq \frac{\lambda}{4}\left\|\Delta^{2} \psi\right\|^{2}+C(\lambda)\|\psi\|_{L^{6}}^{6} \leq \frac{\lambda}{2}\left\|\Delta^{2} \psi\right\|^{2}+C(\lambda,|\Omega|) .
$$

Similarly, we have

$$
\begin{aligned}
& \varepsilon \int_{\Omega} \phi^{2} \Delta^{2} \psi d x \leq \frac{\lambda}{4}\left\|\Delta^{2} \psi\right\|^{2}+C(\varepsilon)\|\phi\|_{L^{4}}^{4}, \leq \frac{\lambda}{8}\left\|\Delta^{2} \psi\right\|^{2}+\frac{1}{2}\|\nabla \phi\|^{2}+C(\varepsilon,|\Omega|), \\
& 2\left(\lambda+\gamma-\frac{\kappa_{3}}{2}\right)\|\Delta \psi\|^{2} \leq \frac{\lambda}{8}\left\|\Delta^{2} \psi\right\|^{2}+C\left(\lambda, \kappa_{3}, \gamma,|\Omega|\right) .
\end{aligned}
$$

From (3.5) and (3.6)-(3.7), we obtain

$$
\begin{aligned}
\Lambda_{3}(\phi, \psi) & \leq-\frac{\lambda}{4}\left\|\Delta^{2} \psi\right\|^{2}-\frac{1}{2}\|\nabla \phi\|^{2}+C_{3}^{*}\left(\gamma, \varepsilon, \lambda, \alpha, \kappa_{3},|\Omega|\right) \\
& \leq C_{3}^{*}\left(\gamma, \varepsilon, \lambda, \alpha, \kappa_{3},|\Omega|\right),
\end{aligned}
$$

that is

$$
\frac{d}{d t}\left(\|\phi\|^{2}+\|\Delta \psi\|^{2}\right)+\kappa_{3}\left(\|\phi\|^{2}+\|\Delta \psi\|^{2}\right) \leq C_{3}^{*} .
$$

Applying the Gronwall inequality, we deduce that

$$
\left(\|\phi\|^{2}+\|\Delta \psi\|^{2}\right) \leq e^{-\kappa_{3} t}\left(\left\|\phi_{0}\right\|^{2}+\left\|\Delta \psi_{0}\right\|^{2}\right)+C_{3}^{*} / \kappa_{3}, \quad \text { for } \quad t \in\left[0, T_{\max }\right) .
$$

Taking $M_{3}=C_{3}^{*} / \kappa_{3}+1$, the proof is completed.

\section{Asymptotic compactness}

Lemma 5. For any given $R>0$ there exists a constant $M_{4}(R)>0$ such that if the initial data $u_{0}=\left(\phi_{0}, \psi_{0}\right)^{T} \in E_{2}$ and $\left\|u_{0}\right\|_{E_{2}}^{2} \leq R$, then $S(t) u_{0}=(\phi, \psi)^{T} \in$ $E_{2}$ for all $t \geq 0$, and

$$
\left\|S(t) u_{0}\right\|_{E_{2}}^{2} \leq M_{4}(R), \quad \text { for } \quad t \geq 0 .
$$

Proof. Take the inner products $\langle(1.1),-2 \Delta \phi\rangle$, and $\left\langle\Delta(1.2),-2 \Delta^{2} \psi\right\rangle$. By the Neumann boundary conditions, we get

$$
\begin{aligned}
& \frac{d}{d t}\|\nabla \phi\|^{2}=-2\|\Delta \phi\|^{2}+2 \alpha\|\nabla \phi\|^{2}-6 \int_{\Omega} \phi^{2}(\nabla \phi)^{2} d x+2 \varepsilon \int_{\Omega} \phi \psi \Delta \phi d x, \\
& \frac{d}{d t}\|\nabla \Delta \psi\|^{2}=-2 \lambda\left\|\nabla \Delta^{2} \psi\right\|^{2}+4 \lambda\left\|\Delta^{2} \psi\right\|^{2}-2(\lambda+\gamma)\|\nabla \Delta \psi\|^{2} \\
& +4 g \int_{\Omega} \psi \nabla \psi \nabla \Delta^{2} \psi d x-6 \int_{\Omega} \psi^{2} \nabla \psi \nabla \Delta^{2} \psi d x-2 \varepsilon \int_{\Omega} \phi \nabla \phi \nabla \Delta^{2} \psi d x .
\end{aligned}
$$


Then summing up the resulting equalities, we see that

$$
\begin{aligned}
& \frac{d}{d t}\left(\|\nabla \phi\|^{2}+\|\nabla \Delta \psi\|^{2}\right)+\kappa_{4}\left(\|\nabla \phi\|^{2}+\|\nabla \Delta \psi\|^{2}\right)=-2\|\Delta \phi\|^{2} \\
& \quad+\left(2 \alpha+\kappa_{4}\right)\|\nabla \phi\|^{2}-6 \int_{\Omega} \phi^{2}(\nabla \phi)^{2} d x+2 \varepsilon \int_{\Omega} \phi \psi \Delta \phi d x-2 \lambda\left\|\nabla \Delta^{2} \psi\right\|^{2} \\
& \quad+4 \lambda\left\|\Delta^{2} \psi\right\|^{2}-2\left(\lambda+\gamma-\frac{\kappa_{4}}{2}\right)\|\nabla \Delta \psi\|^{2}+4 g \int_{\Omega} \psi \nabla \psi \nabla \Delta^{2} \psi d x \\
& \quad-6 \int_{\Omega} \psi^{2} \nabla \psi \nabla \Delta^{2} \psi d x-2 \varepsilon \int_{\Omega} \phi \nabla \phi \nabla \Delta^{2} \psi d x \equiv \Lambda_{4}(\phi, \varphi)
\end{aligned}
$$

Using the Nirenberg inequalities $(n \leq 3)$, we obtain

$$
\begin{aligned}
& \|\nabla \phi\| \leq C_{1}\|\Delta \phi\|^{1 / 2}\|\phi\|^{1 / 2}+C_{2}\|\phi\|, \\
& \|\phi\|_{L^{\infty}} \leq C_{1}\|\Delta \phi\|^{3 / 4}\|\phi\|^{1 / 4}+C_{2}\|\phi\|, \\
& \|\nabla \Delta \psi\| \leq C_{1}\left\|\nabla \Delta^{2} \psi\right\|^{3 / 5}\|\psi\|^{1 / 4}+C_{2}\|\psi\|, \\
& \left\|\Delta^{2} \psi\right\| \leq C_{1}\left\|\nabla \Delta^{2} \psi\right\|^{4 / 5}\|\psi\|^{1 / 4}+C_{2}\|\psi\| .
\end{aligned}
$$

From Lemma 3, and noticing $H^{1}(\Omega) \hookrightarrow L^{4}(\Omega)$ for $n \leq 3$, we deduce that

$$
\begin{aligned}
& 2 \varepsilon \int_{\Omega} \psi \phi \Delta \phi d x \leq \frac{1}{4}\|\Delta \phi\|^{2}+C(\varepsilon)\|\phi\|_{L^{4}}^{4}+C(\varepsilon)\|\psi\|_{L^{4}}^{4} \leq \frac{1}{2}\|\Delta \phi\|^{2}+C(\varepsilon), \\
& 4 \lambda\left\|\Delta^{2} \psi\right\|^{2} \leq \frac{\lambda}{4}\left\|\nabla \Delta^{2} \psi\right\|^{2}+C(\lambda,|\Omega|), \\
& 2\left(\lambda+\gamma-\frac{\kappa_{4}}{2}\right)\|\nabla \Delta \psi\|^{2} \leq \frac{\lambda}{4}\left\|\nabla \Delta^{2} \psi\right\|^{2}+C\left(\lambda, \gamma, \kappa_{4},|\Omega|\right), \\
& 4 g \int_{\Omega} \psi \nabla \psi \nabla \Delta^{2} \psi d x \leq \frac{\lambda}{4}\left\|\nabla \Delta^{2} \psi\right\|^{2}+C(\lambda)\|\psi\|_{L^{4}}^{4}+C(\lambda)\|\nabla \psi\|_{L^{4}}^{4} \\
& \quad \leq \frac{\lambda}{4}\left\|\nabla \Delta^{2} \psi\right\|^{2}+C(\lambda, g,|\Omega|) .
\end{aligned}
$$

Similarly, we obtain

$$
\begin{aligned}
& 6 \int_{\Omega} \psi^{2} \nabla \psi \nabla \Delta^{2} \psi d x \leq \frac{\lambda}{8}\left\|\nabla \Delta^{2} \psi\right\|^{2}+C\|\psi\|_{L 8}^{8}+C\|\nabla \psi\| \leq \frac{\lambda}{4}\left\|\nabla \Delta^{2} \psi\right\|^{2}+C \\
& 2 \varepsilon \int_{\Omega} \phi \nabla \phi \nabla \Delta^{2} \psi d x \leq \frac{\lambda}{4}\left\|\nabla \Delta^{2} \psi\right\|^{2}+C(\lambda, \varepsilon)\|\phi\|_{L^{\infty}}^{2}\|\nabla \phi\|^{2} \\
& \quad \leq \frac{\lambda}{4}\left\|\nabla \Delta^{2} \psi\right\|^{2}+\|\Delta \phi\|+C(\lambda, \varepsilon,|\Omega|)
\end{aligned}
$$

From (4.1) and (4.2)-(4.3), we obtain

$$
\Lambda_{4}(\phi, \varphi) \leq-\frac{3}{4}\|\Delta \phi\|^{2}-\frac{3}{4}\left\|\nabla \Delta^{2} \psi\right\|^{2}+C_{4}^{*}\left(\lambda, \varepsilon, \alpha, \kappa_{4},|\Omega|\right),
$$

that is

$$
\frac{d}{d t}\left(\|\nabla \phi\|^{2}+\|\nabla \Delta \psi\|^{2}\right)+\kappa_{4}\left(\|\nabla \phi\|^{2}+\|\nabla \Delta \psi\|^{2}\right) \leq C_{4}^{*}
$$


Applying the Gronwall inequality, we deduce that

$$
\left(\|\nabla \phi\|^{2}+\|\nabla \Delta \psi\|^{2}\right) \leq e^{-\kappa_{4} t}\left(\left\|\nabla \phi_{0}\right\|^{2}+\left\|\nabla \Delta \psi_{0}\right\|^{2}\right)+\frac{C_{4}^{*}}{\kappa_{4}}, \text { for } t \in\left[0, T_{\max }\right) .
$$

Taking $M_{4}=C_{4}^{*} / \kappa_{4}+1$, the proof is completed.

We now finish the proof of Theorem 1 .

Proof. [Proof of Theorem 1] First, by Lemma 4, the solution semiflow $S(t)_{t>0}$ of reaction-diffusion system (1.1)-(1.2) has a bounded absorbing set $B_{0}$ in $\bar{E}_{1}$. Second, according to Lemma 5 and due to that Sobolev imbedding $E_{2} \hookrightarrow E_{1}$ is compact, this solution semiflow $S(t)_{t \geq 0}$ is asymptotically compact in $E_{1}$, then by Lemma 1 , there exists a global attractor $\mathcal{A}$ for $S(t)_{t \geq 0}$ in $E_{1}$, which is given by $(2.1)$.

\section{The $H^{k}$ global attractor}

In order to consider the global attractor for the system (1.1)-(1.2) in $H^{k}$ space, we introduce the definition as follows:

$$
\begin{aligned}
& H=L^{2}(\Omega), \quad H_{\frac{1}{2}}=\left\{u \in H^{2}(\Omega),\left.\quad \frac{\partial u}{\partial n}\right|_{\partial \Omega}=0\right\}, \\
& H_{1}=\left\{u \in H^{4}(\Omega),\left.\quad \frac{\partial u}{\partial n}\right|_{\partial \Omega}=\left.\frac{\partial \Delta u}{\partial n}\right|_{\partial \Omega}=0\right\} .
\end{aligned}
$$

In this paper, we used to assume that the linear operator

$$
L=-\lambda \Delta^{2}: \quad H_{1} \rightarrow H
$$

is a sectorial operator, which generates an analytic semigroup $e^{t L}$, and $L$ induces the fractional power operators and fractional order spaces as follows

$$
\mathcal{L}^{\alpha}=(-L)^{\alpha}: H_{\alpha} \rightarrow H, \quad(i=1,2), \alpha \in \mathbb{R},
$$

where $H_{\alpha}=D\left(\mathcal{L}^{\alpha}\right)$ is the domain of $\mathcal{L}^{\alpha}$. By the semigroup theory of linear operators, $H_{\beta} \subset H_{\alpha}$ is a compact inclusion for any $\beta>\alpha$. For details of the space $H_{\alpha}$ see [8].

Then, we have the following lemma on the existence of global attractor which is equivalent to Lemma 1 and the proof is similar to $[12,13,14]$.

Lemma 6. Assume that $\left.\left.(\phi(t), \psi(t))=S(t)\left(\phi_{0}, \psi_{0}\right) \quad\left(\left(\phi_{0}, \psi_{0}\right)\right) \in H \times H\right), t \geq 0\right)$ is a solution of (1.1) and $S(t)$ the semigroup generated by (1.1). Assume further that $H_{\alpha}$ is the fractional order space generated by $L$ and

(1) For some $\alpha \geq 0$, there is a bounded set $B \subset H_{\alpha+\frac{1}{4}} \times H_{\alpha+\frac{3}{4}}$, which means that for any $\left(\phi_{0}, \psi_{0}\right) \in H_{\alpha+\frac{1}{4}} \times H_{\alpha+\frac{3}{4}}$, there exists $t_{0} \geq 0$ such that

$$
(\phi(t), \psi(t)) \in B, \forall t>t_{0}
$$


(2) There is a $\beta>\alpha$, such that for any bounded set $U \subset H_{\beta+\frac{1}{4}} \times H_{\beta+\frac{3}{4}}$, there are $T>0$ and $C>0$,

$$
\left\|u\left(t, u_{0}\right)\right\|_{H_{\beta+\frac{1}{4}} \times H_{\beta+\frac{3}{4}}} \leq C, \quad \forall t>T \text { and }\left(\phi_{0}, \psi_{0}\right) \in U .
$$

Then (1.1) has a global attractor $\mathcal{A} \subset H_{\alpha+\frac{1}{4}} \times H_{\alpha+\frac{3}{4}}$ which attracts any bounded set of $H_{\alpha+\frac{1}{4}} \times H_{\alpha+\frac{3}{4}}$ in the $H_{\alpha+\frac{1}{4}} \times H_{\alpha+\frac{3}{4}}$ norm.

For sectorial operators, we also have the following lemma which is important for this paper and can be founded in $[12,13,14]$.

Lemma 7. Assume that $L$ is a sectorial operator which generates an analytic semigroup $T(t)=e^{t L}$. If all eigenvalues $\lambda$ of $L$ satisfy $\operatorname{Re} \lambda<-\lambda_{0}$ for some real number $\lambda_{0}>0$, then for $\mathcal{L}^{\alpha}(\mathcal{L}=-L)$ we have

(1) $T(t): H \rightarrow H_{\alpha}$ is bounded for all $\alpha \in \mathbb{R}$ and $t>0$;

(2) $T(t) \mathcal{L}^{\alpha} x=\mathcal{L} T(t) x, \forall x \in H_{\alpha}$;

(3) For each $t>0, \mathcal{L}^{\alpha} T(t): H \rightarrow H$ is bounded, and

$$
\left\|\mathcal{L}^{\alpha} T(t)\right\| \leq C_{\alpha} t^{-\alpha} e^{-\delta t}
$$

where some $\delta>0$ and $C_{\alpha}>0$ is a constant depending only on $\alpha$;

(4) The $H_{\alpha}$-norm can be defined by $\|x\|_{H_{\alpha}}=\left\|\mathcal{L}^{\alpha} x\right\|_{H}$.

The main result of this paper is given by the following theorem, which provides the existence of global attractors of Eq.(1.1) in any $k$ th space $H^{k}$.

Theorem 2. Assume that $\Omega$ denotes an open bounded domain in $R^{3}$, then for any $k \geq 0$, the initial-boundary value problem (1.1)-(1.2) has a global attractor $\mathcal{A}$ in $\bar{H}^{k} \times H^{k+2}$, and $\mathcal{A}$ attracts any bounded subset of $H^{k} \times H^{k+2}$ in the $H^{k} \times H^{k+2}$-norm.

By Lemma 6, in order to prove Theorem 2, we first prove the following lemma.

Lemma 8. For any $\sigma \geq 0$, the solution $(\phi, \psi)$ of (1.1)-(1.2) is uniformly bounded in $H_{\sigma+\frac{1}{4}} \times H_{\sigma+\frac{3}{4}}$, i.e. for any bounded set $U \subset H_{\sigma+\frac{1}{4}} \times H_{\sigma+\frac{3}{4}}$, there exists $C>0$ such that

$$
\|(\phi, \psi)\|_{H_{\sigma+\frac{1}{4}} \times H_{\sigma+\frac{3}{4}}} \leq C, \forall t>0,\left(\phi_{0}, \psi_{0}\right) \in U \subset H_{\sigma+\frac{1}{4}} \times H_{\sigma+\frac{3}{4}}, \sigma \geq 0 .
$$

Proof. For any $\left(\phi_{0}, \psi_{0}\right) \in L^{2}(\Omega) \times H^{2}(\Omega)$, the solutions $(\phi, \psi)$ of $(1.1)-(1.2)$ can be expressed as

$$
\begin{aligned}
& \phi\left(t, \phi_{0}\right)=e^{t L_{1}} \phi_{0}+\int_{0}^{t} e^{(t-\tau) L_{1}} F_{1}(\phi, \psi) d \tau, \\
& \psi\left(t, \psi_{0}\right)=e^{t L_{2}} \psi_{0}+\int_{0}^{t} e^{(t-\tau) L_{2}} F_{2}(\phi, \psi) d \tau,
\end{aligned}
$$

where $L_{1}=\Delta, L_{2}=-\lambda \Delta^{2}$. By Theorem 1 , there exists attractor in the phase space $L^{2} \times H^{2}$. Using the same way, it is not difficult to prove that there exists attractor in $H^{1} \times H^{3}$, which means $(\phi, \psi) \in H_{\frac{1}{4}} \times H_{\frac{3}{4}}$. 
Step1. We shall prove that for any bounded set $U \subset H_{\sigma+\frac{1}{4}} \times H_{\sigma+\frac{3}{4}}\left(0 \leq \sigma<\frac{1}{4}\right)$, there exists $C>0$ such that

$$
\|(\phi, \psi)\|_{H_{\sigma+\frac{1}{4}} \times H_{\sigma+\frac{3}{4}}} \leq C,\left(\phi_{0}, \psi_{0}\right) \in U \subset H_{\sigma+\frac{1}{4}} \times H_{\sigma+\frac{3}{4}}, 0 \leq \sigma<\frac{1}{4} .
$$

We claim that $F_{2}: H_{\frac{1}{4}} \times H_{\frac{1}{2}} \rightarrow H$ is bound. Based on Lemma 5 and embedding theorem $H^{1} \hookrightarrow L^{6}$ for $n \leq 3$, we have

$$
\begin{aligned}
& \left\|F_{2}(\phi, \psi)\right\|_{H}=\left\|-2 \lambda \Delta \psi-(\gamma+\lambda) \psi+g \psi^{2}-\psi^{3}-\frac{\varepsilon}{2} \phi^{2}\right\|_{H} \\
& \leq C\left(\|\Delta \psi\|_{L^{2}}+\|\psi\|_{L^{4}}^{2}+\|\psi\|_{L^{6}}^{3}+\|\phi\|_{L^{4}}^{2}\right) \leq C\left(\|\psi\|_{H_{\frac{1}{2}}}^{2}+\|\phi\|_{H_{\frac{1}{4}}}^{2}\right) \leq C,
\end{aligned}
$$

where $C$ depends on $\lambda, \gamma, g, \varepsilon,|\Omega|$ but independent of $\phi_{0}$ and $\psi_{0}$. Hence, we obtain

$$
\begin{aligned}
\left\|\psi\left(t, \psi_{0}\right)\right\|_{H_{\sigma+\frac{3}{4}}} & =\left\|e^{t L_{2}} \psi_{0}+\int_{0}^{t} e^{(t-\tau) L_{2}} F_{2}(\phi, \psi) d \tau\right\|_{H_{\sigma+\frac{3}{4}}} \\
& \leq\left\|\psi_{0}\right\|_{H_{\sigma+\frac{3}{4}}}+\left\|\int_{0}^{t} e^{(t-\tau) L_{2}} F_{2}(\phi, \psi) d \tau\right\|_{H_{\sigma+\frac{3}{4}}} \\
& \leq\left\|\psi_{0}\right\|_{H_{\sigma+\frac{3}{4}}}+\int_{0}^{t}\left\|\left(-L_{2}\right)^{\sigma+\frac{3}{4}} e^{(t-\tau) L_{2}}\right\| \cdot\left\|F_{2}(\phi, \psi)\right\|_{H} d \tau \\
& \leq\left\|\psi_{0}\right\|_{H_{\sigma+\frac{3}{4}}}+C \int_{0}^{t}(t-\tau)^{-\left(\sigma+\frac{3}{4}\right)} e^{-\delta(t-\tau)} d \tau \\
& \leq\left\|\psi_{0}\right\|_{H_{\sigma+\frac{3}{4}}}+C \int_{0}^{t} \tau^{-\beta} e^{-\delta \tau} d \tau \\
& \leq C\left\|\left(\phi_{0}, \psi_{0}\right)\right\|_{H_{\sigma+\frac{1}{4}} \times H_{\sigma+\frac{3}{4}}}, \quad\left(0 \leq \sigma<\frac{1}{4}\right)
\end{aligned}
$$

where $\beta=\sigma+\frac{3}{4}$ and $0 \leq \beta<1$.

Similarly, we claim that $F_{1}: H_{\frac{1}{4}} \times H_{\frac{1}{4}} \rightarrow H$ is bound. Based on Lemma 5 and embedding theorem $H^{1} \hookrightarrow L^{6}, H^{1} \hookrightarrow L^{4}$ for $n \leq 3$, we have

$$
\begin{aligned}
& \left\|F_{1}(\phi, \psi)\right\|_{H}=\left\|\alpha \phi-\phi^{3}-\varepsilon \phi \psi\right\|_{H} \\
& \quad \leq C\left(\|\phi\|_{L^{2}}+\|\psi\|_{L^{4}}^{2}+\|\phi\|_{L^{6}}^{3}+\|\phi\|_{L^{4}}^{2}\right) \leq C\left(\|\psi\|_{H_{\frac{1}{4}}}^{2}+\|\phi\|_{H_{\frac{1}{4}}}^{2}\right) \leq C,
\end{aligned}
$$

where $C$ depends on $\alpha, \varepsilon,|\Omega|$ but independent of $\phi_{0}$ and $\psi_{0}$. Hence,

$$
\begin{aligned}
\left\|\phi\left(t, \phi_{0}\right)\right\|_{H_{\sigma+\frac{1}{4}}} & =\left\|e^{t L_{1}} \phi_{0}+\int_{0}^{t} e^{(t-\tau) L_{1}} F_{1}(\phi, \psi) d \tau\right\|_{H_{\sigma+\frac{1}{4}}} \\
& \leq\left\|\phi_{0}\right\|_{H_{\sigma+\frac{1}{4}}}+\left\|\int_{0}^{t} e^{(t-\tau) L_{1}} F_{1}(\phi, \psi) d \tau\right\|_{H_{\sigma+\frac{1}{4}}} \\
& \leq\left\|\phi_{0}\right\|_{H_{\sigma+\frac{1}{4}}}+\int_{0}^{t}\left\|\left(-L_{1}\right)^{2 \sigma+\frac{1}{2}} e^{(t-\tau) L_{1}}\right\| \cdot\left\|F_{1}(\phi, \psi)\right\|_{H} d \tau
\end{aligned}
$$




$$
\begin{aligned}
& \leq\left\|\phi_{0}\right\|_{H_{\sigma+\frac{1}{4}}}+\int_{0}^{t} \tau^{-2 \sigma-\frac{1}{2}} e^{-\delta \tau} d \tau \\
& \leq C\left\|\left(\phi_{0}, \psi_{0}\right)\right\|_{H_{\sigma+\frac{1}{4}} \times H_{\sigma+\frac{3}{4}}}, \quad(0 \leq \sigma<1 / 4),
\end{aligned}
$$

where $0 \leq 2 \sigma+\frac{1}{2}<1$. From (5.2)-(5.3), then (5.1) is proved.

For $\sigma=1 / 8$, we have $\phi \subset H_{3 / 8}$, this meaning $\phi \subset H^{3 / 2}(\Omega)$ (for fractional order Sobolev space). By the embedding theorems of fractional order spaces, we deduce that

$$
\phi(t . x) \subset H^{3 / 2} \hookrightarrow C^{0}(\Omega) \cap H^{1}(\Omega)
$$

Step2. We shall prove that for any bounded set $U \subset H_{\sigma+\frac{1}{4}} \times H_{\sigma+\frac{3}{4}}\left(\frac{1}{4} \leq \sigma<\frac{1}{2}\right)$, there exists $C>0$ such that

$$
\|(\phi, \psi)\|_{H_{\sigma+\frac{1}{4}} \times H_{\sigma+\frac{3}{4}}} \leq C,\left(\phi_{0}, \psi_{0}\right) \in U \subset H_{\sigma+\frac{1}{4}} \times H_{\sigma+\frac{3}{4}}, \frac{1}{4} \leq \sigma<\frac{1}{2} .
$$

We claim that $F_{2}: H_{\frac{1}{4}} \times H_{\frac{3}{4}} \rightarrow H_{\frac{1}{4}}$ is bound. Based on Lemma 5 and embedding theorem $H^{3} \hookrightarrow L^{\infty}$ for $n \leq 3$, we have

$$
\begin{aligned}
& \left\|F_{2}(\phi, \psi)\right\|_{H_{\frac{1}{4}}}=\left\|\nabla\left(-2 \lambda \Delta \psi-(\gamma+\lambda) \psi+g \psi^{2}-\psi^{3}-\frac{\varepsilon}{2} \phi^{2}\right)\right\|_{H} \\
& \left.\quad=\|-2 \lambda \nabla \Delta \psi-(\gamma+\lambda) \nabla \psi+2 g \nabla \psi \psi-3 \psi^{2} \nabla \psi-\varepsilon \phi \nabla \phi\right) \|_{H} \\
& \quad \leq C\left\{\|\nabla \Delta \psi\|_{L^{2}}^{2}+\left(\|\psi\|_{L^{\infty}}^{4}+1\right)\|\nabla \psi\|_{L^{2}}^{2}+\sup _{\Omega}|\phi|^{2}\|\nabla \phi\|_{L^{2}}^{2}\right\} \\
& \quad \leq C\left(\|\psi\|_{H_{\frac{3}{4}}}^{2}+\|\phi\|_{H_{\frac{1}{4}}}^{2}\right) \leq C,
\end{aligned}
$$

where we used (5.4).

$$
\begin{aligned}
& \left\|\psi\left(t, \psi_{0}\right)\right\|_{H_{\sigma+\frac{3}{4}}}=\left\|e^{t L_{2}} \psi_{0}+\int_{0}^{t} e^{(t-\tau) L_{2}} F_{2}(\phi, \psi) d \tau\right\|_{H_{\sigma+\frac{3}{4}}} \\
& \leq\left\|\psi_{0}\right\|_{H_{\sigma+\frac{3}{4}}}+\left\|\int_{0}^{t} e^{(t-\tau) L_{2}} F_{2}(\phi, \psi) d \tau\right\|_{H_{\sigma+\frac{3}{4}}} \\
& \leq\left\|\psi_{0}\right\|_{H_{\sigma+\frac{3}{4}}}+\int_{0}^{t}\left\|\left(-L_{2}\right)^{\sigma+\frac{1}{2}} e^{(t-\tau) L_{2}}\right\| \cdot\left\|F_{2}(\phi, \psi)\right\|_{H_{\frac{1}{4}}} d \tau \\
& \leq\left\|\psi_{0}\right\|_{H_{\sigma+\frac{3}{4}}}+C \int_{0}^{t}(t-\tau)^{-\left(\sigma+\frac{1}{2}\right)} e^{-\delta(t-\tau)} d \tau \\
& \leq\left\|\psi_{0}\right\|_{H_{\sigma+\frac{3}{4}}}+C \int_{0}^{t} \tau^{-\left(\sigma+\frac{1}{2}\right)} e^{-\delta \tau} d \tau \\
& \leq C\left\|\left(\phi_{0}, \psi_{0}\right)\right\|_{H_{\sigma+\frac{1}{4}} \times H_{\sigma+\frac{3}{4}}},(1 / 4 \leq \sigma<1 / 2),
\end{aligned}
$$

where $0 \leq \sigma+\frac{1}{2}<1$.

Similarly, we claim that $F_{1}: H_{\frac{1}{4}} \times H_{\frac{1}{4}} \rightarrow H_{\frac{1}{4}}$ is bound.

$$
\left\|F_{1}(\phi, \psi)\right\|_{H_{\frac{1}{4}}}=\left\|\nabla\left(\alpha \phi-\phi^{3}-\varepsilon \phi \psi\right)\right\|_{H}
$$




$$
\begin{aligned}
& \leq C\left(\|\nabla \phi\|_{L^{2}}^{2}+\|\phi\|_{L^{\infty}}^{4}\|\nabla \phi\|_{L^{2}}^{2}+\|\phi\|_{L^{\infty}}^{2}\|\nabla \psi\|_{L^{2}}^{2}+\|\psi\|_{L^{\infty}}^{2}\|\nabla \phi\|_{L^{2}}^{2}\right) \\
& \leq C\left(\|\psi\|_{H_{\frac{1}{4}}}^{2}+\|\phi\|_{H_{\frac{1}{4}}}^{2}\right) \leq C,
\end{aligned}
$$

where $C$ depends on $\alpha, \varepsilon,|\Omega|$ but independent of $\phi_{0}$ and $\psi_{0}$. Therefore

$$
\begin{aligned}
& \left\|\phi\left(t, \phi_{0}\right)\right\|_{H_{\sigma+\frac{1}{4}}}=\left\|e^{t L_{1}} \phi_{0}+\int_{0}^{t} e^{(t-\tau) L_{1}} F_{1}(\phi, \psi) d \tau\right\|_{H_{\sigma+\frac{1}{4}}} \\
& \leq\left\|\phi_{0}\right\|_{H_{\sigma+\frac{1}{4}}}+\left\|\int_{0}^{t} e^{(t-\tau) L_{1}} F_{1}(\phi, \psi) d \tau\right\|_{H_{\sigma+\frac{1}{4}}} \\
& \leq\left\|\phi_{0}\right\|_{H_{\sigma+\frac{1}{4}}}+\int_{0}^{t}\left\|\left(-L_{1}\right)^{2 \sigma} e^{(t-\tau) L_{1}}\right\| \cdot\left\|F_{1}(\phi, \psi)\right\|_{H_{\frac{1}{4}}} d \tau \\
& \leq\left\|\phi_{0}\right\|_{H_{\sigma+\frac{1}{4}}}+\int_{0}^{t} \tau^{-2 \sigma} e^{-\delta \tau} d \tau \\
& \leq C\left\|\left(\phi_{0}, \psi_{0}\right)\right\|_{H_{\sigma+\frac{1}{4}} \times H_{\sigma+\frac{3}{4}}},(1 / 4 \leq \sigma<1 / 2),
\end{aligned}
$$

where $0 \leq 2 \sigma<1$. From (5.6)-(5.7), then (5.5) is proved.

Lemma 9. For any $\sigma>0$, (1.1)-(1.2) has a bounded absorbing set in $H_{\sigma+\frac{1}{4}} \times$ $H_{\sigma+\frac{3}{4}}$. That is, for any bounded set $U \subset H_{\sigma+\frac{1}{4}} \times H_{\sigma+\frac{3}{4}}$ there are $T>0$ and $a$ constant $C>0$ independent of $\phi_{0}$ and $\psi_{0}$, such that

$$
\|(\phi, \psi)\|_{H_{\sigma+\frac{1}{4}} \times H_{\sigma+\frac{3}{4}}} \leq C, \forall t>T,\left(\phi_{0}, \psi_{0}\right) \in U \subset H_{\sigma+\frac{1}{4}} \times H_{\sigma+\frac{3}{4}}, \sigma \geq 0 .
$$

Proof. Step1. We shall show that for any $0 \leq \sigma<\frac{1}{4}$, (1.1)-(1.2) has a bounded absorbing set in $H_{\sigma+\frac{1}{4}} \times H_{\sigma+\frac{3}{4}}$. The solution $(\phi, \psi)$ can be expressed as

$$
\begin{aligned}
& \phi\left(t, \phi_{0}\right)=e^{(t-T) L_{1}} \phi\left(T, \phi_{0}\right)+\int_{T}^{t} e^{(t-\tau) L_{1}} F_{1}(\phi, \psi) d \tau, \\
& \psi\left(t, \psi_{0}\right)=e^{(t-T) L_{2}} \psi\left(T, \psi_{0}\right)+\int_{T}^{t} e^{(t-\tau) L_{2}} F_{2}(\phi, \psi) d \tau .
\end{aligned}
$$

On the other hand, note that

$$
\left\|e^{t L_{1}}\right\| \leq C e^{-d \lambda_{1} t}, \quad\left\|e^{t L_{2}}\right\| \leq C e^{-d \lambda_{1}^{2} t},
$$

where $\lambda_{1}>0$ is the first eigenvalue of the equation

$$
-\Delta u=\lambda u,\left.\quad u\right|_{\partial \Omega}=0 .
$$

By assertion (1) of lemma 7, for any given $T>0$ and $0 \leq \sigma<\frac{1}{4}$, we have

$$
\left\|e^{(t-T) L_{i}} \psi\left(T, \psi_{0}\right)\right\|_{H_{\sigma}} \rightarrow 0, \quad i=1,2, \quad \text { as } t \rightarrow \infty .
$$

Using assertion (3) of lemma 7, we have

$$
\left\|\psi\left(t, \psi_{0}\right)\right\|_{H_{\sigma+\frac{3}{4}}}=\left\|e^{(t-T) L_{2}} \psi\left(T, \psi_{0}\right)+\int_{T}^{t} e^{(t-\tau) L_{2}} F_{2}(\phi, \psi) d \tau\right\|_{H_{\sigma+\frac{3}{4}}}
$$




$$
\begin{aligned}
& \leq\left\|e^{(t-T) L_{2}} \psi\left(T, \psi_{0}\right)\right\|_{H_{\sigma+\frac{3}{4}}}+\left\|\int_{T}^{t} e^{(t-\tau) L_{2}} F_{2}(\phi, \psi) d \tau\right\|_{H_{\sigma+\frac{3}{4}}} \\
& \leq\left\|e^{(t-T) L_{2}} \psi\left(T, \psi_{0}\right)\right\|_{H_{\sigma+\frac{3}{4}}}+\int_{T}^{t}\left\|\left(-L_{2}\right)^{\sigma+\frac{3}{4}} e^{(t-\tau) L_{2}}\right\| \cdot\left\|F_{2}(\phi, \psi)\right\|_{H} d \tau \\
& \leq\left\|e^{(t-T) L_{2}} \psi\left(T, \psi_{0}\right)\right\|_{H_{\sigma+\frac{3}{4}}}+C \int_{T}^{t}(t-\tau)^{-\left(\sigma+\frac{3}{4}\right)} e^{-\delta(t-\tau)} d \tau \\
& \leq\left\|e^{(t-T) L_{2}} \psi\left(T, \psi_{0}\right)\right\|_{H_{\beta}}+C \int_{T}^{t} \tau^{-\beta} e^{-\delta \tau} d \tau \leq C,
\end{aligned}
$$

where $\beta=\sigma+\frac{3}{4}, 0 \leq \beta<1, C>0$ is a constant independent of $\psi_{0}$. Similarly, we have

$$
\begin{aligned}
& \left\|\phi\left(t, \phi_{0}\right)\right\|_{H_{\sigma+\frac{1}{4}}}=\left\|e^{(t-T) L_{1}} \phi\left(T, \phi_{0}\right)+\int_{T}^{t} e^{(t-\tau) L_{1}} F_{1}(\phi, \psi) d \tau\right\|_{H_{\sigma+\frac{1}{4}}} \\
& \quad \leq\left\|e^{(t-T) L_{1}} \phi\left(T, \phi_{0}\right)\right\|_{H_{\sigma+\frac{1}{4}}}+\left\|\int_{T}^{t} e^{(t-\tau) L_{1}} F_{1}(\phi, \psi) d \tau\right\|_{H_{\sigma+\frac{1}{4}}} \\
& \quad \leq\left\|e^{(t-T) L_{1}} \phi\left(T, \phi_{0}\right)\right\|_{H_{\sigma+\frac{1}{4}}}+\int_{T}^{t}\left\|\left(-L_{1}\right)^{2 \sigma+\frac{1}{2}} e^{(t-\tau) L_{1}}\right\| \cdot\left\|F_{1}(\phi, \psi)\right\|_{H} d \tau \\
& \quad \leq\left\|e^{(t-T) L_{1}} \phi\left(T, \phi_{0}\right)\right\|_{H_{\sigma+\frac{1}{4}}}+\int_{T}^{t} \tau^{-2 \sigma-\frac{1}{2}} e^{-\delta \tau} d \tau \leq C,
\end{aligned}
$$

where $0 \leq 2 \sigma+\frac{1}{2}<1, C>0$ is a constant independent of $\phi_{0}$.

Step2. We shall show that for any $\frac{1}{4} \leq \sigma<\frac{1}{2}$, (1.1)-(1.2) has a bounded absorbing set in $H_{\sigma} \times H_{\sigma+\frac{3}{4}}$.

$$
\begin{aligned}
& \left\|\psi\left(t, \psi_{0}\right)\right\|_{H_{\sigma+\frac{3}{4}}}=\left\|e^{(t-T) L_{2}} \psi\left(T, \psi_{0}\right)+\int_{T}^{t} e^{(t-\tau) L_{2}} F_{2}(\phi, \psi) d \tau\right\|_{H_{\sigma+\frac{3}{4}}} \\
& \leq\left\|e^{(t-T) L_{2}} \psi\left(T, \psi_{0}\right)\right\|_{H_{\sigma+\frac{3}{4}}}+\left\|\int_{T}^{t} e^{(t-\tau) L_{2}} F_{2}(\phi, \psi) d \tau\right\|_{H_{\sigma+\frac{3}{4}}} \\
& \quad \leq\left\|e^{(t-T) L_{2}} \psi\left(T, \psi_{0}\right)\right\|_{H_{\sigma+\frac{3}{4}}}+\int_{T}^{t}\left\|\left(-L_{2}\right)^{\sigma+\frac{1}{2}} e^{(t-\tau) L_{2}}\right\| \cdot\left\|F_{2}(\phi, \psi)\right\|_{H_{\frac{1}{4}}} d \tau \\
& \leq\left\|e^{(t-T) L_{2}} \psi\left(T, \psi_{0}\right)\right\|_{H_{\sigma+\frac{3}{4}}}+C \int_{T}^{t}(t-\tau)^{-\left(\sigma+\frac{1}{2}\right)} e^{-\delta(t-\tau)} d \tau \\
& \leq\left\|e^{(t-T) L_{2}} \psi\left(T, \psi_{0}\right)\right\|_{H_{\sigma+\frac{3}{4}}}+C \int_{T}^{t} \tau^{-\left(\sigma+\frac{1}{2}\right)} e^{-\delta \tau} d \tau \\
& \leq\left\|e^{(t-T) L_{2}} \psi\left(T, \psi_{0}\right)\right\|_{H_{\sigma+\frac{3}{4}}}+C
\end{aligned}
$$

where $C>0$ is a constant independent of $\psi_{0}$.

$$
\begin{gathered}
\left\|\phi\left(t, \phi_{0}\right)\right\|_{H_{\sigma+\frac{1}{4}}}=\left\|e^{(t-T) L_{1}} \phi\left(T, \phi_{0}\right)+\int_{T}^{t} e^{(t-\tau) L_{1}} F_{1}(\phi, \psi) d \tau\right\|_{H_{\sigma+\frac{1}{4}}} \\
\leq\left\|e^{(t-T) L_{1}} \phi\left(T, \phi_{0}\right)\right\|_{H_{\sigma+\frac{1}{4}}}+\left\|\int_{T}^{t} e^{(t-\tau) L_{1}} F_{1}(\phi, \psi) d \tau\right\|_{H_{\sigma+\frac{1}{4}}}
\end{gathered}
$$




$$
\begin{aligned}
& \leq\left\|e^{(t-T) L_{1}} \phi\left(T, \phi_{0}\right)\right\|_{H_{\sigma+\frac{1}{4}}}+\int_{T}^{t}\left\|\left(-L_{1}\right)^{2 \sigma} e^{(t-\tau) L_{1}}\right\| \cdot\left\|F_{1}(\phi, \psi)\right\|_{H_{\frac{1}{4}}} d \tau \\
& \leq\left\|e^{(t-T) L_{1}} \phi\left(T, \phi_{0}\right)\right\|_{H_{\sigma+\frac{1}{4}}}+\int_{T}^{t} \tau^{-2 \sigma} e^{-\delta \tau} d \tau \\
& \leq\left\|e^{(t-T) L_{1}} \phi\left(T, \phi_{0}\right)\right\|_{H_{\sigma+\frac{1}{4}}}+C
\end{aligned}
$$

by iteration, we can obtain (5.8).

Proof. [Proof of Theorem 2.] By Lemma 8 and Lemma 9, we immediately conclude the proof of Theorem 2 is completed.

\section{Conclusions}

Based on the regularity estimates for the semigroups and the classical existence theorem of global attractors, we prove that the system possesses a global attractor in the space $H_{k+\frac{1}{4}} \times H_{k+\frac{3}{4}}$. Comparing this paper with $[12,13,14]$. The system (1.1)-(1.2) is a two-component model. We define the product Hilbert spaces, using the Lumer-Phillips theorem and the generation theorem for analytic semigroups. The main difficulties for treating the problem (1.1)-(1.2) are caused by the nonlinearity of low order terms, and linear higher order terms are not homogeneous. The existence of the attractor in $H^{k} \times H^{k+2}$, guarantee a solution of the model equations for any value of the control parameters. This explains the following: 1) the solutions are robust and not sensitive to changes in the value of its control parameters and 2) the diversity of patterns that explain different biological systems (pigmentation vertebrate) and inhere animals (membranes porous medium and ternary mixtures with surfactants).

\section{Acknowledgement}

The authors would like to thanks the referees' valuable suggestions for the revision and improvement of the manuscript. This work is supported by the Jilin Scientific and Technological Development Program (No. 20170101143JC).

\section{References}

[1] J.W. Cholewa and T. Dlotko. Global attractor for the CahnHilliard system. Bull. Austral. Math. Soc., 49(2):277-292, 1994. https://doi.org/10.1017/S0004972700016348.

[2] E.J. Crampin and P.K. Maini. Reaction-diffusion models for biological pattern formation. Mathods Appl. Anal., 8(3):415-428, 2001. https://doi.org/10.4310/maa.2001.v8.n3.a3.

[3] A. Doelman, T.J. Kaper and Paul A. Zegeling. Pattern formation in the one-dimensional Gray-Scott model. Nonlinearity, 10(2):523-563, 1997. https://doi.org/10.1088/0951-7715/10/2/013.

[4] A. Eden and V.K. Kalantarov. 3d convective Cahn-Hilliard equation. Commun. Pure Appl. Anal., 6(4):1075-1086, 2007. https://doi.org/10.3934/cpaa.2007.6.1075. 
[5] D. Henry. Geometric theory of semilinear parabolic equations. Springer-Verlag, Berlin-New York, 1981. https://doi.org/10.1007/BFb0089647.

[6] D. Li and C. Zhong. Global attractor for the Cahn-Hilliard system with fast growing nonlinearity. J. Differential Equations, 149(2):191-210, 1998. https://doi.org/10.1006/jdeq.1998.3429.

[7] C. Liu and A. Liu. The existence of global attractor for a sixth order parabolic equation. Politehn. Univ. Bucharest Sci. Bull. Ser. A Appl. Math. Phys., 76(1):115-128, 2014.

[8] T. Ma and S. Wang. Stability and bifurcation of nonlinear evolution equations. Science Press, Beijing, (In Chinese), 2006.

[9] M.A. Morales, J.F. Rojas, J. Oliveros and A.A. Hernández S. A new mechanochemical model: Coupled Ginzburg-Landau and Swift-Hohenberg equations in biological patterns of marine animals. Journal of Theoretical Biology, 368:37-54, 2015. https://doi.org/10.1016/j.jtbi.2014.12.005.

[10] M.A. Morales, J.F. Rojas, I. Torres and E. Rubio. Modeling ternary mixtures by mean-field theory of polyelectrolytes: Coupled GinzburgLandau and Swift-Hohenberg equations. Physica A, 391(3):779-791, 2012. https://doi.org/10.1016/j.physa.2011.08.054.

[11] G.R. Sell and Y. You. Dynamics of Evolutionary Equations. Springer-Verlag, New york, 2002. https://doi.org/10.1007/978-1-4757-5037-9.

[12] L. Song, Y. He and Y. Zhang. The existence of global attractors for semilinear parabolic equation in $H^{k}$ spaces. Nonlinear Analysis, 68(11):3541-3549, 2008. https://doi.org/10.1016/j.na.2007.03.045.

[13] L. Song, Y. Zhang and T. Ma. Global attractor of the Cahn-Hilliard equation in $H^{k}$ spaces. J. Math. Anal. Appl., 355(1):53-62, 2009. https://doi.org/10.1016/j.jmaa.2009.01.035.

[14] L. Song, Y. Zhang and T. Ma. Global attractor of a modified SwiftHohenberg equation in $H^{k}$ spaces. Nonlinear Anal., 72(1):183-191, 2010. https://doi.org/10.1016/j.na.2009.06.103.

[15] R. Temam. Infinite-dimensional Dynamical Systems in Mechanics and Physics. Springer-Verlag, 1997. https://doi.org/10.1007/978-1-4612-0645-3.

[16] H. Wu and S.M. Zheng. Global attractor for the 1-d thin film equation. Asymptot. Anal., 51(2):101-111, 2007.

[17] Y. You. Asymptotical dynamics of Selkov equations. Disc. Contin. Dyn. Systems, Series S, 2(1):193-219, 2009.

[18] Y. You. Dynamics of three-component reversible Gray-Scott model. Disc. Contin. Dyn. Systems, Series B, 14(4):1671-1688, 2010.

[19] Y. You. Asymptotic dynamics of reversible cubic autocatalytic reactiondiffusion systems. Commun. Pure Appl. Anal., 10(5):1415-1445, 2011. https://doi.org/10.3934/cpaa.2011.10.1415. 